\title{
Advancing Cross-Scale Understanding of Reactive Fluid Induced Chemo- Morphological Changes in Multi-Phase Environments using X-Ray and Neutron Scattering Measurements
}

\author{
Greeshma Gadikota, ${ }^{1 *}$ Meishen Liu, ${ }^{1}$ and Hassnain Asgar ${ }^{1}$ \\ ${ }^{1}$ Department of Civil \& Environmental Engineering \\ University of Wisconsin, Madison \\ Email: gadikota@wisc.edu; Phone: 857-253-8724
}

\begin{abstract}
One of our grand societal challenges lies in meeting our rising energy demand while reducing detrimental environmental impacts which include managing greenhouse gas emissions and efficiently using and treating our water resources. However, the development of sustainable subsurface energy technologies such as water efficient hydrocarbon recovery and geologic carbon storage need to be grounded in a fundamental scientific understanding of dynamic reactive fluid-induced chemo-morphological changes in heterogeneous materials representative of subsurface environments. Latest advancements in multi-scale X-ray scattering which encompass Ultra-Small, Small, and Wide Angle X-Ray Scattering (USAXS/SAXS/WAXS) and very Small Angle Neutron Scattering (vSANS) measurements combined with complementary analytical techniques now allow us to dynamically determine the structural and morphological changes as geochemically active fluids interact with materials representative of surface environments. In this context, we evaluate the influence of reactive fluids (e.g., acidic gases such as $\mathrm{CO}_{2}$ ) on the chemical and morphological changes in nanoporous materials (e.g., shales), and map the influence of temperature on dynamic changes in the porosity and the chemical composition of shales using multiscale X-ray and neutron scattering and tomography measurements. These studies highlight the need to account for the influence of reactivity on the dynamic changes in the pore structures of subsurface nanoporous solids in Darcy's description of flow through porous media. Further, rising need to enhance the permeability in subsurface environments using water-efficient approaches has called for designing novel materials. We discuss the design and characterization of $\mathrm{CO}_{2}$-responsive phase changing nanofluids with the ability to form hydrogels to induce fractures in subsurface environments. These fluids are constructed from silica nanoparticles and poly(allylamine) (PAA) as the building blocks. The enhanced development of $\mathrm{CO}_{2}$-induced coiled networks in these novel nanofluids was captured using in-operando Ultra Small Angle $\mathrm{X}$-Ray Scattering, and the changes in the bonding mechanisms leading to the formation of reversible hydrogels are inferred from Fourier Transform-Infrared Spectroscopy measurements. These research efforts highlight the need to advance cross-scale scientific understanding of reactive fluid induced chemomorphological changes in complex heterogeneous materials using advanced scattering techniques.
\end{abstract}

